\title{
Optical biochemical and chemical sensors
}

\author{
Laura M. Lechuga
}

Published online: 6 October 2012

(C) Springer-Verlag Berlin Heidelberg 2012

Ongoing changes in the analytical field are demanding novel technological diagnostic tools that could facilitate quick, accurate, reliable, and cost-effective testing, avoiding the expensive, complex, and time-consuming procedures typically employed in standard analytical evaluation. The results of those tests open the way to implement appropriate actions in time, leading to an improved socioeconomic outcome. Optical biochemical and chemical sensors have positioned themselves as one of the best options to be such novel technological diagnostic tools. The unique characteristics of optical sensing, such as its inherently high sensitivity, compactness, reliability, electromagnetic immunity, easy interconnectivity, possibility of remote sensing, wide detection range using a minimum volume of sample and reagents, and low cost, have promoted it as the preferred solution. The main applications of this technology will be in clinical diagnostics, but the technology will be beneficial in other impactful fields, such as genomics, proteomics, pharmacology, health care, environmental monitoring, food analysis, agriculture, intracellular sensing, defense, and security.

Optical biochemical and chemical sensors have experienced exponential growth in the last decade thanks to strong interdisciplinary participation by academia and industry. Developments have been stimulated by a very active and productive research community with a mind to apply its research to solve real-world analytical problems. Optical biochemical and chemical sensors require broad multidisciplinary knowledge with special emphasis on the needs of end users. In recent years, we have seen how an open communication has been established between academic researchers and companies. As a

Published in the special paper collection Optical Biochemical and Chemical Sensors with guest editor Laura M. Lechuga.

L. M. Lechuga $(\bowtie)$

Research Center on Nanoscience and Nanotechnology (CIN2)

CSIC and CIBER-BBN,

ETSE, Campus UAB-Edificio Q, 2nd Floor, QC/2113, Bellaterra, 08193 Barcelona, Spain

e-mail: laura.lechuga@cin2.es result, several of these optical sensor technologies have been successfully commercialized, but there is still a long way to go to cover all the stringent requirements for each particular field of application. Full technological transfer and commercialization of the most mature optical sensor knowledge still faces significant technological hurdles.

In recent years, emerging technologies combining new design concepts such as microstructured fibers, localized surface plasmon resonance sensors, nanoparticle-based sensing, miniaturized spectroscopic systems, and integrated silicon photonic sensors such as microring resonators, photonic crystals, silicon nanowires, slot waveguides, nanopillars, and novel interferometric devices have shown a strong impetus. The coming years will be crucial for making great advances in the discovery of new sensing mechanisms, their technological transfer into commercial products, and the demonstration of real-life applications. The dream of having a device in the palm of our hand able to deliver diagnostics instantly could become a reality very soon and will certainly impact our daily life.

This special issue is devoted to the latest innovations in the dynamic area of optical biochemical and chemical sensing, showing both cutting-edge concepts and key emerging trends. Although the focus is on optical detection and transducing mechanisms, topics in the fields of innovative surface functionalization protocols, real-world applications, system integration, miniaturization and automation, and multiplexed detection are also discussed.

Recent advances in optical detection and transducing mechanisms are considered in several contributions, such as those covering sensors based on ring-shaped organic photodiodes, nanoplasmonic arrays, or silicon photonic sensors. The utilization of new luminescent materials for gas sensing and the fabrication of nanoparticles which could potentially replace single dye labels to provide enhanced assay performance in point-of-care biomedical devices are also discussed. Other articles reflect the increasing interest in miniaturization and portability of sensor instruments capable of measuring 
several analytes simultaneously or the recent trends in integration of microfluidics with optical sensors as is the case of digital microfluidics. Improved biofunctionalization strategies for emerging silicon photonic integrated sensors are also covered. To encourage user confidence and aid in future acceptance, some of the contributions are dedicated to reallife applications in clinical and environmental fields.

This special issue should be a valuable source of information for all readers with an interest in chemical and biochemical sensing. I would like to thank all the authors for their valuable contributions to this special issue, the referees for their prompt responses and critical but constructive comments, and the Editorial Office for its professionalism and effective cooperation during the preparation of the issue.

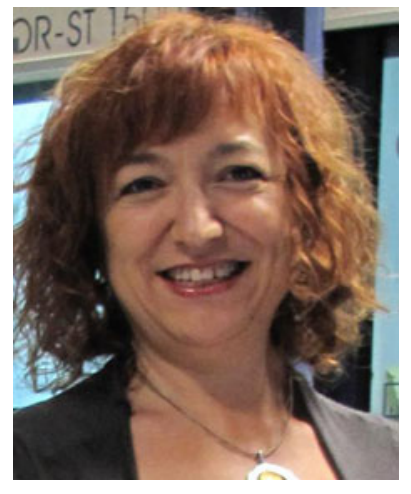

Laura M. Lechuga is Head of the Nanobiosensors and Bioanalytical Applications Group at the Center on Nanoscience and Nanotechnology (CIN2, CSIC) in Barcelona (Spain). Her main research interests are the development of biosensor devices based on plasmonics, magnetoplasmonics, silicon photonics, and nanomechanics principles, including surface biofunctionalization, microfluidics, and labon-a-chip integration and their application in diagnostics. She has published over 150 original articles and has eight families of patents, and has participated in the establishment of two spin-offs. 